\title{
Correction to: Did You Find It on the Internet? Ethical Complexities of Search Engine Rankings
}

\author{
Cansu Canca
}

\section{Correction to:}

Chapter 19 in: H. Werthner et al. (eds.), Perspectives on Digital Humanism, https://doi.org/10.1007/978-3-030-86144-5_19

The original version of the chapter was inadvertently published with an error. The affiliation of the author Cansu Canca has now been corrected to "AI Ethics Lab, Cambridge, MA, USA". 